\title{
Interprétation Philosophique du Métabolisme Comme Moment de Restauration de la Dignité de la vie chez Hans Jonas
}

\author{
Traoré Grégoire, \\ Département de Philosophie, Université Alassane Ouattara, Côte d'Ivoire
}

Doi:10.19044/esj.2019.v15n29p79

URL:http://dx.doi.org/10.19044/esj.2019.v15n29p79

\section{Résumé}

La présente étude s'inscrit dans le vaste champ de la philosophie de la biologie de Hans Jonas dont le but est d'appréhender les caractéristiques essentielles de la vie afin de lui donner tout le sens et la valeur qu'elle mérite. Une approche herméneutique et holistique de la vie n'est possible, selon Jonas, qu'en procédant à une interprétation philosophique du système métabolique afin de permettre à la vie de recouvrer la dignité qu'elle a perdue avec la réification du vivant suscitée par les théories modernes de la biologie, le dualisme cartésien et l'anthropocentrisme occidental. À partir d'une démarche critique, herméneutique et phénoménologique, il s'agit pour nous et dans cette étude, de montrer que seule une maîtrise de l'essence du vivant permet d'agir adéquatement à son égard et ce, dans un contexte axiologique. Cependant, en fondant son éthique sur une ontologie, Jonas adopte une position fondamentaliste, intégriste et conservatrice qui s'écarte des normes éthiques du monde contemporain fondées sur des principes démocratiques.

Mots-clés: Axiologie, Biologie, Dignité, Métabolisme, Organisme, Responsabilité, Physico-chimique, Vivant 


\title{
Philosophical Interpretation of Metabolism as a Moment of Restoration of the Dignity of Life at Hans Jonas
}

\author{
Traoré Grégoire, \\ Département de Philosophie, Université Alassane Ouattara, Côte d'Ivoire
}

\begin{abstract}
The present study is part of the broad field of Hans Jonas' philosophy of biology whose purpose is to grasp the essential characteristics of life in order to give it all the meaning and value it deserves. According to Jonah, a hermeneutic and holistic approach to life is only possible through a philosophical interpretation of the metabolic system in order to allow life to recover the dignity it has lost with the reification of the living created by the modern theories of biology, Cartesian dualism and Western anthropocentrism. Based on a critical, hermeneutical and phenomenological approach, it is for us and in this study, to show that only a mastery of the essence of the living can act appropriately for it in an axiological context. . However, by basing his ethic on an ontology, Jonah adopts a fundamentalist, fundamentalist and conservative stance that departs from the ethical norms of the contemporary world based on democratic principles.
\end{abstract}

Keywords: Axiology, Biology, Dignity, Metabolism, Organism, Responsibility, Physicochemical, Living

\section{Introduction}

L'éthique de la responsabilité de Hans Jonas s'enracine dans une ontologie qui s'appuie sur un phénomène biologique dont les caractéristiques sont amplement présentées dans Le phénomène de la vie (1966), Le principe responsabilité (1979) et Évolution et liberté (2000). Il s'agit du phénomène du métabolisme en tant qu'ensemble des transformations physico-chimiques qui s'effectuent dans les tissus de l'organisme vivant à travers les dépenses énergétiques, les échanges, la nutrition, etc. Ce mode d'organisation et de fonctionnement qui assure son équilibre, sa permanence et sa survie est la vie elle-même.

Appréhendant la vie exclusivement à travers ses caractéristiques morphologique et chimique, la biologie moderne, selon Jonas, n'en restitue pas tout le sens. Bien qu'elle permette de saisir une certaine dimension de la 
vie, la biologie moderne est incapable de comprendre le vivant dans sa totalité. Dans le contexte de la modernité au centre duquel se trouve la philosophie dualiste de Descartes, l'objectivité est devenue le maître-mot de la science cherchant toujours à prouver son efficacité pratique. À cette fin, toutes les approches gnoséologiques valorisant des aspects non matériels de l'être vivant sont refoulées comme éloignant la raison du savoir objectif ou comme le pense E. Kant, du savoir auquel il a accès selon les structures et les pouvoirs de sa propre nature, de son entendement. Seuls aspects phénoménaux de la vie sont soumis aux normes d'objectivité de la science autorisant à leur ôter toute dignité propre, donc les privant du droit au respect.

Les conséquences des pratiques techniques actuelles qui se font au détriment de la nature sont vues par H. Jonas comme émanant de cette forme d'explication des phénomènes du monde qui repose sur une césure entre l'homme et les autres phénomènes de la nature. Le monde est donc en crise en raison du regard que la science contemporaine fait projeter sur les phénomènes de la vie, c'est-à-dire en raison de la non prise en compte de la dignité naturelle des êtres humains ou non. Il a besoin d'un regard nouveau ou d'un recadrage de la compréhension que l'homme s'en fait. Comment y parvenir ? Ou encore, comment l'homme peut-il arriver à restaurer la valeur absolue de la vie ?

C'est pour répondre à cette interrogation qu'une évaluation holistique et philosophique du phénomène du métabolisme, dans la perspective ouverte par Jonas, mérite d'être entreprise, car elle seule est susceptible de lui conférer toute sa valeur. D'ailleurs comme le pense Jonas, une explication philosophique du vivant rend obsolète la séparation instaurée entre l'âme et le corps depuis le dualisme cartésien.

Les concepts de liberté, d'identité, d'ipséité, de subjectivité et de téléologie qu'on retrouve dans l'approche jonassienne du vivant, manifestent l'idée que celui-ci renferme une intériorité révélatrice d'une dimension axiologique à partir de laquelle doivent se construire toutes les normes régulatrices de l'action humaine à l'égard de la nature. Ainsi, toutes les activités humaines qui tiennent compte du sens du métabolisme de l'organisme, en tant que vecteur de compréhension du caractère moniste de la vie, deviennent respectueuses de la nature. On peut donc dire que le métabolisme constitue la norme naturelle à partir de laquelle toutes les activités doivent se modeler. Il s'agit d'exploiter la dynamique métabolique en tant que modèle expressif de la transversalité de la lutte du vivant pour se maintenir en vie comme moyen d'instauration de l'équilibre symbiotique nécessaire à la continuité de l'existence sur terre, et surtout en tant que voie de restauration de la dignité de la vie.

En réfléchissant sur les implications éthiques du phénomène du métabolisme, nous avons pour ambition de dégager une détermination 
biologique et métaphysique qui servira de base à une interprétation éthique et philosophique dudit phénomène.

L'interprétation que nous voulons entreprendre se fera en trois moments et ce, dans une démarche critique, herméneutique et phénoménologique. Dans la première phase de notre travail, nous mettrons en lumière le caractère biologique et physico-chimique du métabolisme (1). Par la suite, nous dévoilerons l'analyse métaphysique qu'en donne Hans Jonas (2) pour, enfin, mettre en avant, la dimension axiologique de cette dynamique, à première vue, biologique (3).

\section{Approche biologique et physico-chimique du métabolisme}

Le métabolisme est un phénomène biologique important dans l'explication du vivant. Toute la vie se réduit à son fonctionnement si bien que tous les êtres vivants sont considérés comme des systèmes métaboliques. Desagher (1998, p. 13), dans son livre intitulé Métabolisme, fonde ses réflexions sur la chimie organique qui permet d'appréhender le fonctionnement du métabolisme. Celui-ci (1998, p. 25) le définit comme « l'ensemble des réactions chimiques se déroulant dans un système vivant et permettant à celui-ci de récupérer l'énergie dont il a besoin, de construire les molécules le constituant, de détruire les molécules en excès, inutiles et même parfois toxiques ». Ces différentes réactions sont des chaînons métaboliques dont chacune est catalysée par une enzyme spécifique correspondant à une étape d'un métabolisme.

Le métabolisme repose sur deux types d'enzymes. Ils sont des substances protéiniques qui facilitent ou accroissent toute réaction biochimique dans un corps vivant : il y a d'une part, le catabolisme dont le rôle est de dégrader et d'éliminer les molécules actives in vivo, après la récupération de l'énergie dont elles disposent et d'autre part, l'anabolisme qui sert, quant à lui, à synthétiser les molécules. Cependant, il faut faire remarquer que l'anabolisme et le catabolisme sont des processus chimiques interactifs, dans lesquels interviennent les mêmes séquences métaboliques.

Le métabolisme permet, en effet, de retracer les déférentes transformations chimiques de molécules organiques. Selon Desagher (1998, p. 19), il est impossible de « comprendre le métabolisme si on ne connaît pas les principales règles régissant la structure et la réactivité des molécules ». D'ailleurs, la connaissance du métabolisme exige de comprendre les différents mécanismes par lesquels l'enzyme transforme en produit, le substrat après sa fixation. La conception du métabolisme s'inscrit purement dans un domaine physico-chimique qui constitue les différentes réactions chimiques opérées dans l'organisme vivant. Ces réactions qui ne se réalisent qu'à travers des entités vivantes, sont la marque distinctive de tout système vivant. Le maintien 
du métabolisme dans des conditions adéquates participe à la santé ou à l'harmonie organique de l'être vivant.

C'est pourquoi, Matt Stone (2014, p. 8) dans son livre Mangez et fondez remarque que « le maintien d'un haut niveau de production d'énergie cellulaire avec un haut taux métabolique et des niveaux modérés d'exposition aux hormones de stress est le meilleur des atouts pour la santé ». Il considère cette idée comme une vérité fondamentale. Pour lui, le métabolisme est fondé sur la régulation de la concentration des liquides se trouvant dans le corps. Il soutient que la nourriture et les liquides que nous absorbons peuvent soit accroître soit réduire la concentration des fluides de notre corps. Les aliments que nous apportons au corps, sont biologiquement importants dans le contrôle et le bon fonctionnement de notre système organique. Ainsi, lorsque le corps fonctionne normalement, il est à l'abri de toutes sortes de maladies.

Le principe du métabolisme que présente Matt Stone est essentiellement basé sur la santé organique du vivant elle-même fonction de la qualité de la vie. La qualité de la nutrition y tient le rôle primordial. Selon lui, « il s'agit uniquement d'un aspect significatif du système corporel que vous pouvez modifier en plus d'autres pratiques de base que vous trouverez réalistes et durables et qui améliorent votre qualité de vie générale » (Stone, 2014, p.11). Cette conception de Stone du métabolisme axée sur la santé par la nutrition (diététique) pourrait être également expliquée sur le plan sportif. Le métabolisme, dans la conception de Stone, dépend de la manière dont l'être vivant se nourrit. Pour augmenter son métabolisme, il faut, selon lui, avoir une alimentation qualitativement équilibrée. Serge Desagher s'inscrit dans la même logique mécanique du métabolisme que Stone. Mais, à la différence de Stone qui met l'accent sur la biologie alimentaire, il se consacre à l'étude du fonctionnement du métabolisme.

Pour Hans Jonas (2005a, pp. 25-26), cette conception quasi mécanique du phénomène du métabolisme ne saurait restituer tout le sens de la vie. Elle présente le vivant comme un amas de chair mû par un enchaînement de règles physiques extérieures. Ce que vise Jonas comme objectif, c'est de montrer qu'en appréhendant le vivant à partir de ses déterminations extérieures, la biologie moderne a tendance à négliger la dimension d'intériorité centrale à la vie. Cette approche purement matérialiste du vivant, rend la vie plus difficile à cerner qu'elle ne l'était. L'explication de la vie à partir de ses éléments physiques, matériels, au lieu d'aboutir à une connaissance objective, translucide de la vie, rend son explication plus opaque et obscure. Pour Jonas, il est inconséquent que les dimensions de l'intériorité et de l'extériorité de la vie soient séparées dès lors qu'elles sont deux réalités biologiques fusionnées. C'est pourquoi, il suggère qu'une lecture philosophique renouvelée de la biologie peut reconquérir la dimension intérieure qui est la part la mieux connue du vivant afin de comprendre l'organisme, et de restituer ainsi à l'unité 
psychophysique de la vie la place dans l'ensemble qu'elle a perdue depuis le dualisme de Descartes.

Dans sa structure, la vie est marquée par des contradictions telles que la créativité et la moralité, la liberté et la nécessité, etc., qui sont présentes dans ses formes les plus élémentaires. Cette bipolarité est originairement présente dans tout être vivant. Elle est un aspect essentiel de la vie qu'il convient de comprendre dans toute sa complexité en tant qu'elle attribue, par ricochet, au vivant les qualités du sentir et du percevoir, du mouvement et du désir, etc. Cette approche que Jonas accorde à la vie, en général, éloigne sa pensée de la conception anthropocentrique de la philosophie occidentale, des considérations objectivantes et matérialistes de la biologie scientifique pour faire du vivant, un être dont la dimension de l'intériorité exige une compréhension plus approfondie. D’ailleurs, pour Jonas, la séparation consacrée entre l'extériorité et intériorité depuis Descartes est arbitraire, car il y a une unité psychophysique naturelle entre l'âme et le corps qui permet de « reconnaître le caractère originaire de la relation existant entre ma vie et les autres vies, en deçà de tout partage de la réalité entre l'objectif et le subjectif » (E. Pommier, 2013a, p. 63). Cette réalité est perceptible dans la vie et peut être suivie au cours de son évolution qui, selon la métaphysique de Jonas, traverse ses différentes organisations telles que les facultés et les fonctions biologiques qui se complexifient au fur et à mesure qu'on tend vers le vivant le plus évolué, à savoir l'être humain.

\section{Approche métaphysique et vitaliste de la vie chez Hans Jonas}

Tout se passe comme si chez Hans Jonas, l'être humain est l'être le plus évolué émanant du processus d'évolution de la vie. À la lumière de la compréhension du sujet humain, il devient donc possible de comprendre le reste de la vie. Depuis l'époque antique, plusieurs théories du vivant se sont succédées (le fixisme, le vitalisme, le mécanisme et l'évolutionnisme). Elles avaient pour but de comprendre, d'expliquer ou de maitriser le fonctionnement et les structures du vivant.

S'opposant à toutes conceptions mécaniste et objectivante de la vie qui ne rendent pas compte de son sens fondamental, Jonas remarque que certaines fonctions spécifiques de l'être ou possibilités naturelles telles que la liberté, l'autonomie, la dépendance, existent dans les premières formes de la vie. Pour lui, « les grandes contradictions que l'être humain découvre en lui-même liberté et nécessité, autonomie et dépendance, moi et monde, relation et isolement, créativité et mortalité ont leur préfiguration déjà en germe dans les formes primitives de la vie » (H. Jonas, 2005a, p. 26). Ce sont là, des capacités cognitives naturelles qui, du point de vue de Jonas, s'expriment au niveau du vivant. Autrement dit, le métabolisme est le point de départ de toute expression de la liberté et même de la vie. La liberté se manifeste à travers le percevoir et 
l'agir et plus précisément au niveau de l'esprit et de la volonté. Pour Jonas, «le métabolisme, couche fondamentale de toute existence organique, manifeste une liberté - mieux, qu'il est lui-même la première forme de liberté »(H. Jonas, 2005a, p. 28). La liberté apparait dans les structures organiques du vivant.

Ce qui est remarquable dans une telle affirmation c'est que la liberté n'est pas forcément liée à une détermination mentale et psychologique. Elle atteste la volonté de l'organisme vivant d'affirmer son être par rapport au reste du monde même si son existence se retrouve engagée dans une tension entre être et non-être » au regard de l'inévitable phénomène de la létalité. Cette tension qui menace de rompre son équilibre prouve plutôt l'existence d'une fragilité immanente à l'être. Pourtant, ce n'est pas cette fragilité qui détermine profondément l'organisme, mais la lutte permanente qu'elle lui impose de s'affirmer sur le non-être. Cette lutte permet à l'organisme d'entretenir constamment une relation avec la matière pour se maintenir en vie. En effet, l'être vivant est éminemment dépendant de la matière dont le niveau de disponibilité a une incidence sur son mode d'existence et même sur la possibilité effective de cette existence. Cela signifie que la liberté dont jouit le vivant est limitée par la présence des moyens de subsistance dont la disponibilité constante est incertaine. Dès lors, pour Jonas (2005a, p.30), «balançant ainsi entre être et non-être, l'organisme n'a la possession de son être que de manière conditionnelle et révocable ».

La rareté des ressources naturelles peut donc entraîner l'anéantissement du vivant. Le métabolisme intègre l'idée que le non-être est une possibilité de l'être vivant. Être, c'est déjà être ouvert à la possibilité du non-être. Dans son autoaffirmation, l'être vivant lutte continuellement contre le non-être qui peut surgir ou revenir dans le champ physique de l'être, à tout moment. En dehors des causes accidentelles et naturelles, l'indisponibilité des ressources matérielles se présente comme un facteur qui met en péril sa survie. Bien que l'être vivant renouvelle son identité dans un rapport constant qu'il entretient avec la matière, il est confronté au non-être. C'est pourquoi, «que la vie soit mortelle est certes sa contradiction fondamentale, mais comme une partie inséparable de son essence, sans laquelle elle n'est même pas pensable » (H. Jonas, 2005a, p.31). Tout vivant est mortel parce que la mort est inhérente à son être. Cependant, dans cette lutte perpétuelle contre le non-être, la vie finit par s'incliner devant la mort, le non-être.

$\mathrm{Au}$ regard de tout ce qui précède, il apparaît évident que chez Jonas, le vivant dispose d'une intériorité dont le dynamisme se voit à travers ses différentes réactions face au monde extérieur. Toutefois, aux dires de Jonas (2005a, p.34) « (...) si l'intériorité est coextensive à la vie, alors ne peut suffire une interprétation purement mécaniste du vivant, c'est-à-dire une interprétation selon les seuls concepts de l'extériorité ». Si l'intériorité est 
considérée comme une dimension caractéristique du vivant, alors toute explication de la vie qui se fait uniquement à partir de ses déterminations quantitatives ou extérieures est erronée. En fait, «pour pouvoir interpréter comme signe d'une intériorité ce qui se donne à l'extérieur, il faut déjà saisir l'extériorité comme expression d'une intériorité et donc être en rapport à elle » (E. Pommier, 2013b, p.48.). Ce qui implique qu'une approche purement matérialiste du vivant ne restitue pas tout le sens de l'intériorité de la vie.

D’ailleurs, le métabolisme montre que certains fonctionnements du vivant renforcent son unité psychophysique. À ce propos, Jonas souligne que, malgré les échanges de matière que le vivant a avec le monde, il conserve son identité, son soi. C'est un individu qui se démarque de tous les autres et qui demeure le même à travers le temps. Néanmoins, la conservation de son identité ne fait pas perdre au vivant son caractère transitif qui se manifeste dans le métabolisme. Pour Jonas, le système vivant est marqué par le métabolisme parce qu'en tant que cause qui détermine le devenir de l'organisme, il est le mode le plus expressif de la vie. Le métabolisme est essentiellement la vie dont les diverses manifestations la distinguent de la machine. En revanche, désigner un corps vivant en tant que «système métabolique », c'est admettre indirectement que le système lui-même est complètement et permanemment le produit de son activité métabolisante et en plus qu'aucune partie de cette activité ne cesse d'être la manifestation du métabolisme lui-même. C'est pour cette raison qu'il est erroné de comparer tout organisme à une machine comme l'a soutenu Descartes.

Éric Pommier (2013b) présente une position conciliatrice entre Descartes et Jonas vis-à-vis de la thèse de l'unité psychophysique du vivant. À travers cette position, il montre que Descartes ne s'était pas véritablement démarqué de cette conception du vivant puisqu'il est possible de déceler dans certaines de ses œuvres des points de vue qui pourraient s'inscrire dans la perspective du monisme intégral. À la lumière de ce qui précède, on pourrait donc dire que la théorie cartésienne s'inscrit dans une perspective méthodologique et non comme une vérité ontologique. En s'inscrivant dans cette conception du vivant, il paraît loisible de soutenir que le métabolisme traduit non seulement cette unité du vivant longtemps récusée par la philosophie, mais aussi il est l'expression même de la vie.

Le système métabolique est le résultat d'une activité qui permet à l'être vivant d'une part, de se maintenir en vie, et d'autre part, de se reproduire, de croître et de réagir aux stimili du monde extérieur. Bien qu'il exprime une liberté qui est rattachée à son être, le vivant manifeste une dépendance vis-àvis de la matière. « Pour pouvoir échanger de la matière, la forme vivante doit en avoir à sa disposition, et elle la trouve en dehors d'elle, dans le « monde » étranger. Ainsi, la vie est elle-même tournée vers le monde dans une relation particulière de sujétion et de pouvoir » (H. Jonas, 2005a, p.45). C'est un besoin 
dont la satisfaction pousse le vivant à se diriger vers le monde extérieur pourvoyeur d'éléments nutritifs. Cet échange suppose la quête de moyens que le vivant trouve dans le monde extérieur. La forme vivante, pour sa propre satisfaction, la continuité et la conservation de son être, se tourne constamment vers le monde extérieur en tant que milieu regorgeant de tous les produits en termes d'aliments ou d'énergie pour sa survie. Dans cette dépendance fondamentale vis-à-vis du monde extérieur, il doit en principe découler toutes les normes qui doivent réguler l'intervention de l'homme dans la nature. La théorie écologique de Jonas est un biocentrisme qui trouve sa consistance dans cette idée montrant que la disponibilité et la qualité des ressources naturelles conditionnent la survie de l'humanité et de tout être vivant.

Ce caractère biocentrique montre que l'organisme est mû par une cause finale qui conditionnerait son évolution. Pour Jonas (2005b, p. 99), « il n’y a pas d'organisme sans téléologie ; il n'y a pas de téléologie sans intériorité ; et la vie ne peut être connue que par la vie ». Cela signifie que toute entité vivante dispose d'une intériorité dont la finalité est de maintenir la vie. Elle se définit par rapport à une cause finale (le telos) qui est la marque d'une volonté des organismes vers un seul but : leur parfaite réalisation et par conséquent, leur propre vie.

Ainsi, dans la mesure où la vie est l'ensemble de ce qui s'exécute de soi-même et suivant une finalité, cela implique qu'elle ne peut s'appréhender comme une simple machine ou un objet. Pour Jonas, des faits particuliers de l'organisme, tant à l'extérieur qu'à l'intérieur, permettent de comprendre son unité psychophysique comme une réalité sensible. Tous ces faits qui réalisent son identité matérielle et corporelle, à travers le temps, sont perceptibles dans le métabolisme. Dans son livre autobiographique Souvenirs, Jonas montre que l'essence de la vie se dévoile véritablement à travers les manifestations concrètes du vivant. Cependant, le vivant renferme quelque chose qui ne peut être réduit à sa dimension purement corporelle. Dans le même ouvrage, Jonas (2005c, p.239) indique que ce sont-là, les raisons pour lesquelles, « une philosophie de l'organique conduit nécessairement à une éthique ». C'est donc sur la base de cette philosophie organique qu'il élabore son éthique de la responsabilité. Celle-ci met en œuvre les principes et les normes pour une protection efficiente de la nature au compte de l'existence des générations présentes et à venir.

\section{Le métabolisme comme source axiologique de la vie}

Tout dans la nature en tant qu'environnement des êtres vivants, concourt au bien-être de la vie. Tous les êtres vivants cherchent perpétuellement d'une manière ou d'une autre, leur propre épanouissement. C'est une quête qui n'est pas effectuée de manière arbitraire puisqu'elle est universellement ancrée dans leur nature. Elle est une tendance légitime chez 
tous les êtres vivants. À partir du moment où cette aspiration se révèle comme un droit naturel, pour Jonas, son respect devient une obligation pour les êtres vivants évolués, notamment pour les êtres humains. Il le dit précisément dans Le principe responsabilité, en ces termes : «mais alors en résulterait également l'obligation - autrement dit malgré tout un devoir - de respecter ce droit dans les autres, donc de ne pas l'entraver, peut-être même de le promouvoir » (Jonas, 1993, p.110.).

$\mathrm{Au}$ niveau social, la quête du bonheur est un droit universellement légitime que tout être humain a l'obligation de respecter vis-à-vis des autres. En respectant ce droit chez les autres, nous favorisons indirectement le nôtre. Pour Jonas, le visage de l'autre dépasse le cadre de la société humaine. Il est aussi celui de chacun des êtres qui animent la biosphère. En établissant l'analogie avec la réalité sociale, il montre que seule une solidarité anthropobiocosmique peut restaurer l'équilibre symbiotique de la nature. Par ailleurs, en entretenant des fins, la nature montre qu'elle n'est pas axiologiquement neutre. Avec l'attestation que la nature agit selon une finalité et que cette finalité est même contenue dans la nature des choses, nous gagnons quelque chose pour l'éthique qui a besoin de la validité objective des valeurs qui précisément pour cela doivent devenir des fins. Par le fait d'avoir des fins la nature met tout en en œuvre pour les légitimer. À partir de ces considérations axiologiques, Hans Jonas trouve l'opportunité de fonder, en raison, l'éthique sur une ontologie.

Jonas (1993, p.111) explique justement « que le monde ait des valeurs, cela découle certes directement du fait qu'il a des fins (...) ». Autrement dit, le simple fait que le monde entretienne des fins, suppose également qu'il renferme des valeurs. Il existe donc des valeurs qui sont rattachées au monde. En découvrant des valeurs intrinsèques à la nature, Jonas pense avoir fait sauter le dernier verrou de la philosophie occidentale qui récusait l'idée que l'éthique puisse avoir ses repères dans une ontologie, dans une théorie de l'être. À partir du moment où la nature contient en elle des valeurs ou ses propres valeurs, il appartient désormais aux hommes de les respecter. Ce devoir ontologique est également renforcé par l'action anthropique qui fragilise davantage son environnement naturel.

Cependant, il faut faire remarquer qu'il existe chez Jonas un fort désir de rapprocher l'être humain des autres êtres vivants. Il existe chez lui un certain anthropomorphisme qui pourrait être justifié par les aspects philosophiques du darwinisme. L'évolutionnisme, sans le vouloir, consacrait l'idée d'un monisme (l'unité psychophysique) de l'être vivant au détriment de l'homme qui voit sa position centrale dans la nature remise en cause. L'âme, l'esprit et la matière deviennent des entités qui appartiennent à l'entièreté du vivant. De la sorte, Jonas pense que, l'évolutionnisme darwinien a détruit la position privilégiée de l'humain, laquelle avait donné sa signature pour un 
traitement cartésien, purement matériel, de tout le reste. Ainsi, la continuité de l'évolution, qui rattache désormais l'homme au monde animal, interdisait de considérer «son esprit, et de manière générale les phénomènes spirituels, comme l'irruption soudaine, en ce point précis du fleuve de la vie dans sa totalité ; d'un principe ontologiquement étranger » (H. Jonas, 2005a, p.33).

Avec la théorie darwinienne, le dualisme cartésien perd de sa valeur. On peut même soutenir, à partir de là, qu'avec l'évolutionnisme, la dernière citadelle du dualisme, c'est-à-dire l'isolement de l'homme du reste de la nature s'écroule. L'homme recouvre la disposition de sa propre évidence intime pour donner sens au monde auquel il appartient. Les bases de l'anthropocentrisme s'effondrent avec l'évolutionnisme. À partir de cet instant, il est possible de considérer qu'il existe une proximité entre l'homme et les autres êtres vivants. Ainsi, le darwinisme a érodé la conception cartésienne de l'homme et de la nature plus efficacement qu'aucune critique métaphysique n'avait réussie à le faire. Mais, « dans l'indignation bruyante qui s'est élevée contre l'atteinte à la dignité de l'homme par la doctrine de son origine animale, on n'a pas vu qu'en vertu du même principe, c'était la totalité du monde de vivant qui recevait quelque chose de la dignité de l'homme » (H. Jonas, 2005a, p.33-34).

Cette dignité qui est uniquement concédée à l'être humain, s'étend désormais à la totalité du vivant. L'évolutionnisme restaure la dignité du vivant qu'elle a perdu avec l'anthropocentrisme qui établissait une rupture profonde entre le règne animal et le règne humain. Dorénavant, la nature ne peut être étrangère à l'homme puisque « la nature qui a produit un être agissant en fonction de fins ne peut elle-même être étrangère à la finalité » (M-G. Pinsart, 2002, p. 146.). Ontologiquement, chez Jonas, il n'y a pas de différence de nature entre l'homme et l'animal, mais simplement une différence de degré. Les attributs de l'homme tels que la sensation, l'aspiration, la souffrance, la jouissance s'étendent à tous les vivants.

$\mathrm{Au}$ cours des différentes étapes de l'évolution du vivant, l'on peut remarquer la manifestation des sentiments subjectifs tels que la peur, l'angoisse et l'effort qui apparaissent à travers sa conservation physique. L'argument de Jonas qui permet de légitimer ce rapport de parenté entre l'animal et l'être humain s'origine dans la théorie de Charles Darwin selon laquelle l'homme descendrait du singe. Cette preuve hypothétique donne de suggérer l'idée que l'homme et l'animal présentent des caractéristiques communes. Pour Jonas, «c'est seulement de ce point de vue qu'on pourrait montrer qu'en s'attachant à des valeurs, la nature a également l'autorité de les sanctionner et qu'elle est habilitée à exiger leur reconnaissance de nous et de tout vouloir en son sein » (H. Jonas, 1993, p.113).

Cependant, cet acte de reconnaissance des valeurs immanentes à la nature relève-t-elle de la volonté ou du consentement de l'homme ? Cet acte de reconnaissance requiert-il absolument l'assentiment de l'être humain ? La 
théorie des fins qui débouche sur celle des valeurs est considérée par Jonas comme le moment de la réponse à cette importante question. Il existe chez Jonas un lien entre l'être et le devoir. Car, le bien réside dans ce qui a de la valeur, « pour autant qu'il est de son propre fait et non du fait d'un désir, d'un besoin ou d'un choix, est justement, d'après son concept, ce dont la possibilité contient l'exigence de sa réalité et ce qui devient ainsi un devoir à condition qu'existe une volonté capable de percevoir l'exigence et de la traduire en agir » (H. Jonas, 1993, p.115).

Ce n'est pas une décision qui provient d'une volonté personnelle, mais de la revendication intérieure d'un bien en soi qui revendique sa propre effectivité. C'est une décision qui procède du sujet agissant et qui est contenu dans sa nature. Cela implique que la théorie de l'être peut devenir le fondement de la théorie éthique chez Jonas. En entretenant des fins ou en poursuivant des buts, la nature pose des valeurs qui ne doivent absolument pas être compromises ou détruites par l'action humaine. En d'autres termes, entraver cette aspiration légitime de la nature peut être considérée comme une irresponsabilité de la part de l'homme. Réunir les conditions de réalisation d'un tel bien est donc un acte de responsabilité de l'être humain.

Il faut faire remarquer que dans cette tendance de la vie vers cette fin, il existe une autoaffirmation fondamentale de l'être qui se présente comme préférable au non-être. Cette préférence de l'être par rapport à cette fin effective est la preuve que la valeur poursuivie par celui-ci est au-dessus de toutes les valeurs. C'est un bien ou une valeur qu'il désire absolument. Cet intérêt que les êtres visent peut être également empêché par l'être humain en tant qu'un être potentiellement destructeur grâce au pouvoir que lui procure son savoir technoscientifique. Pour ce faire, il est appelé à intégrer dans son action cet intérêt des êtres d'exister dans toute leur plénitude. Il doit dépasser ses intérêts égoïstes pour entreprendre des actions qui favorisent l'accomplissement de la fin naturelle que recherchent tous les êtres. L'homme doit mettre en œuvre les moyens adéquats pour parvenir à cette fin. D'ailleurs, le caractère fragile de l'être doit aiguiser le sens de la responsabilité de l'homme à son égard.

\section{Conclusion}

L'étude sur le métabolisme et ses implications éthiques, nous a permis de comprendre que ledit phénomène, en tant qu'expression de l'essence de l'être vivant, constitue le fondement de la philosophie de la vie de Jonas. L'approfondissement et l'enrichissement de cette thématique, dévoile que la vie dans son effectivité est caractérisée par son unité physico-psychique faisant qu'elle ne peut être traitée comme un simple objet. Comprendre le métabolisme, c'est comprendre que la vie dans sa complexité tend vers une finalité qu'il faut absolument respecter. 
En poursuivant des fins, la vie pose des valeurs. Cette conception biocentrique du monde chez Jonas atteste que la nature a une dignité découverte à travers les différentes manifestations du vivant. Par ailleurs, si l'homme est considéré comme le principal responsable de la dégradation des ressources naturelles, c'est parce que de tous les êtres vivants, il est non seulement celui qui fait preuve d'une grande voracité, mais aussi celui dont les actions dans la nature sont extrêmement dévastatrices. La responsabilité à l'égard de la nature, le creuset même de la dignité de la vie, est ontologique.

Cependant, il faut faire remarquer qu'en fondant l'éthique sur une ontologie, Jonas semble adopter une position fondamentaliste qui suscite un rejet de toute forme de démocratie et de toute éthique qui émanerait de la volonté humaine. Une telle éthique peut-elle subsister dans une société contemporaine multiculturaliste marquée par des principes démocratiques? Cette préoccupation que l'on retrouve chez Franco Volpi, Gilbert Hottois et MarieGeneviève Pinsart montre que l'éthique de la responsabilité de Jonas pourrait être confrontée à de véritables problèmes quant à sa mise en œuvre pragmatique.

\section{References:}

1. DESAGHER Serge, 1998, Métabolisme, approche physico-chimique, Paris, Éditions Ellipses,

2. HOTTOIS Gilbert (dir.), 1993, Hans Jonas. Nature et responsabilité, Paris, Éditions J. Vrin.

3. HOTTOIS Gilbert (éd.), 1993, Aux fondements d'une éthique contemporaine. H. Jonas et H. T. Engelhardt, Paris, Éditions J. Vrin.

4. JONAS Hans, 1993, Le principe responsabilité, une éthique pour la civilisation technologique, traduit de l'allemand par Jean Greish, Paris, Éditions du Cerf.

5. JONAS Hans, 1998, Pour une éthique du futur, traduit de l'allemand par Sabine Cornille et Philippe Ivernel, Paris, Éditions Payot et Rivages.

6. JONAS Hans, 2005a, Évolution et liberté, traduit Philippe Ivernel et Sabine Cornille, Paris, Éditions Payot et rivages.

7. JONAS Hans, 2005b, Le phénomène de la vie, vers une biologie philosophique, traduit de l'anglais par Danielle Lories, Bruxelles, Éditions De Boeck.

8. JONAS Hans, 2005c, Souvenirs, traduit de l'allemand par Sabine Cornille et Philippe Ivernel, Paris, Éditions Payot et Rivages.

9. KANT Emmanuel, 1972, Fondements de la métaphysique des mœurs, Paris, Éditions Victor Delbos. 
10. PINSART Marie-Geneviève, 2002, Jonas et la liberté, dimensions théologiques, ontologiques, éthiques et politiques, Paris, Éditions J. Vrin.

11. POMMIER Éric, 2013a, Hans Jonas, Paris, Éditions Les Belles Lettres.

12. POMMIER Éric, 2013b, Ontologie de la vie et éthique de la responsabilité selon Hans Jonas, Paris, Éditions J. Vrin.

13. STONE Matt, 2014, Mangez et fondez, comment augmenter son métabolisme par la nutrition, traduit de l'anglais américain par Martin Verbeke, Éditions Archangel Ink. 